\title{
Geraniol and Linalool Loaded Nanoemulsions and Their Antimicrobial Activity
}

\author{
Igori BALTA ${ }^{1 \dagger}$, Lucian BRINZAN ${ }^{1 \dagger}$, Alexandros Ch. STRATAKOS ${ }^{2}$, Mark LINTON ${ }^{2}$, Carmel KELLY ${ }^{2}$, Laurette \\ PINKERTON $^{2}$, Nicolae CORCIONIVOSCHI ${ }^{2 *}$ \\ ${ }^{1}$ University of Agricultural Sciences and Veterinary Medicine Cluj-Napoca. Faculty of Animal Science \\ and Biotechnologies. Cluj-Napoca, Romania \\ ${ }^{2}$ Agri-Food and Biosciences Institute, Bacteriology Branch, Belfast, United Kingdom. \\ *Corresponding author, e-mail: Nicolae.Corcionivoschi@afbini.gov.uk \\ ${ }^{\dagger}$ Equal contribution
}

Bulletin UASVM Animal Science and Biotechnologies 74(2)/ 2017

Print ISSN 1843-5262; Electronic ISSN 1843-536X

DOI:10.15835/buasvmcn-asb: 0025

\begin{abstract}
Geraniol and linalool have been found to be effective against foodborne microorganisms in vitro. However, due to their hydrophobic nature, it is difficult to achieve an even dispersion in foods with high water content resulting in dramatic loss of activity. The aim of the study was to fabricate geraniol or linalool nanoemulsions and investigate their effect against Escherichia coli K12, Listeria innocua and Pseudomonas lundensis in a meat simulation medium. The agar diffusion assay revealed that both geraniol and linalool had a potent antimicrobial activity against all bacteria. Dynamic light scattering showed that geraniol and linalool nanoemulsions had a mean diameter of $68.22 \pm 2.46$ and $173.59 \pm 4.15 \mathrm{~nm}$, respectively. Killing assay results showed that both nanoemulsions were able to significantly reduce E. coli and L. innocua counts by approx. $3 \mathrm{log} \mathrm{CFU} / \mathrm{ml}$. Ps. lundensis proved to be more resistant to both nanoemulsions showing a reduction of approx. $1.2 \log \mathrm{CFU} / \mathrm{ml}$,. Overall, this study showed that nanoemulsions loaded with geraniol or linalool represent a promising antimicrobial system to improve food preservation and food safety.
\end{abstract}

Keywords: antimicrobial, geraniol, linalool, nanoemulsion

\section{INTRODUCTION}

Foodborne contamination has posed a major public health concern worldwide for a very long time (Settanni et al., 2012). In the U.S.A., the number of foodborne illnesses is estimated to about 5 million reported cases that lead towards 60,000 hospitalizations and 1800 deaths and about half of foodborne outbreaks are linked to the consumption of contaminated meat (Magnus M., 2008). Meat and meat products are prone to microbial spoilage during slaughtering, processing and storage because they possess an ideal nutrient matrix that can favour the growth of pathogenic and spoilage microorganisms. Essential oils have been gaining importance as food preservatives as many studies have found that they possess significant antimicrobial properties against a broad range of foodborne pathogens (Zhang et al., 2016). These substances are considered "natural" and their use for commercial applications is very attractive since many consumers are now concerned about the addition of synthetic compounds to foods (Burt, 2004). Geraniol, the main component of rose oil, palmarosa oil, and citronella oil and linalool the main compounds of coriander essential oil have been found to have antimicrobial activity in vitro (Burt, 2004). However, due to their hydrophobic nature, it is difficult to achieve an even dispersion in foods with high water content which results in dramatic loss of activity. Different antimicrobial delivery systems have been proposed to improve effectiveness of antimicrobials in food matrices by increasing their dispersion and protecting them from coming in to contact with food components (e.g. proteins, lipids) and losing their activity (Xiao et al., 2011; Zahi et al., 2015). A potential 
Tab 1. Antibacterial activity of pure geraniol and linalool against $E$. coli $\mathrm{K} 12, L$. innocua and P. lundensis. Values represent the diameter of inhibition zone in $\mathrm{mm} \pm$ standard deviation.

\begin{tabular}{cccc}
\hline Bacteria & E.coli K12 & Ps. lundensis & L. innocua \\
\hline Geraniol & $11.78 \pm 0.55 \mathrm{~mm}$ & $12.50 \pm 0.93 \mathrm{~mm}$ & $13.83 \pm 0.35 \mathrm{~mm}$ \\
\hline Linalool & $18.32 \pm 1.17 \mathrm{~mm}$ & $14.55 \pm 1.14 \mathrm{~mm}$ & $16.50 \pm 2.11 \mathrm{~mm}$ \\
\hline
\end{tabular}

alternative to current recommended methods is the use of emulsified essential oils (Landry et al., 2014). This method has recently been reported to be suitable for application in the food industry for fabricating effective antimicrobial nanoemulsions from essential oils (Chang et al., 2013). Based on these findings and the fact that geraniol and linalool are good antimicrobial agents, the emulsification method was used to produce nanoemulsions. The purpose of this study was to characterise the geraniol and linalool nanoemulsions produced and investigate their effectiveness against Escherichia coli K12, Listeria innocua, Pseudomonas lundensis in a meat simulation medium at different time intervals.

\section{MATERIALS AND METHODS}

\section{Cultures}

Escherichia coli K12, Listeria innocua and Pseudomonas lundensis were cultured for $24 \mathrm{~h}$ in brain-heart infusion broth at 37,37 and $30^{\circ} \mathrm{C}$, respectively.

\section{Disc diffusion assay}

Antimicrobial activity was evaluated by the disc diffusion method according by measuring the diameter of the inhibition zone around the disc (6 $\mathrm{mm}$ ) for each of the different antimicrobial agents.

\section{Formation of antimicrobial nanoemulsions}

The preparation of the antimicrobial nanoemulsion was based on a method previously reported by Chang et al., (2013). Geraniol or Linalool (4 g) (Sigma-Aldrich) was added to $6 \mathrm{~g}$ of medium chain triglyceride (MCT) oil (Miglyol 812, Witten, Germany) and thoroughly mixed for $5 \mathrm{~min}$ at 125 RPM. Once mixed, Tween $80^{\circledR}(10 \mathrm{~g})$ (SigmaAldrich) was added to the oil mixture and mixed for another $5 \mathrm{~min}$ at $125 \mathrm{RPM}$. The oil/Tween 80 mixture $(20 \mathrm{~g})$ was titrated, at a rate of $2 \mathrm{~mL} / \mathrm{min}$, into $80 \mathrm{~g}$ of $5.0 \mathrm{mM}$ sodium citrate buffer ( $\mathrm{pH} 3.4$ ) containing a magnetic stirring bar set to $600 \mathrm{RPM}$ and allowed to mix for an additional $15 \mathrm{~min}$. The emulsion was filter sterilized through a sterile
$0.22 \mu \mathrm{m}$ filter and stored in sterile $50 \mathrm{~mL}$ tubes at $2-5{ }^{\circ} \mathrm{C}$ for up to 1 week.

\section{Characterisation of nanoemulsions}

Droplet size was measured using dynamic light scattering (Zetasizer Nano ZS, Malvern Instruments, UK). This instrument determines the particle size from intensity-time fluctuations of a laser beam $(633 \mathrm{~nm})$ scattered from a sample at an angle of $173^{\circ}$.

\section{Killing assay}

Nanoemulsions at a geraniol and linalool concentration of $4000 \mathrm{ppm}$ were prepared in sterilized meat simulation medium $(20 \mathrm{~g}$ of bacteriological peptone, $16 \mathrm{~g}$ of Lab Lemco, $8 \mathrm{~g}$ of yeast extract, $0.2 \mathrm{~g}$ of $\mathrm{MgSO}_{4} \cdot 7 \mathrm{H}_{2} \mathrm{O}, 0.038 \mathrm{~g}$ of $\mathrm{MnSO}_{4} \cdot \mathrm{H}_{2} \mathrm{O}, 1 \mathrm{ml}$ of Tween $80,5 \mathrm{~g}$ of lactic acid (sterilized separately), $40 \mathrm{~g}$ of $\mathrm{NaCl}$. Overnight E. coli K12, L. innocua and Ps. lundensis cultures in BHI broth were washed once in PBS and inoculated at $5 \log \mathrm{CFU} / \mathrm{ml}$ to the corresponding nanoemulsion solutions, mixed and incubated at 37,37 and $30^{\circ} \mathrm{C}$ statically for $0,4 \mathrm{~h}$, and $24 \mathrm{~h}$. The survival of all bacteria at a specific nanoemulsion and incubation time was enumerated by serial dilution and plating onto TSAYE agar. Control treatment did not contain any nanoemulsion.

\section{Statistical analysis}

The experiment was performed three times on different occasions in order to obtain three independent replicates. The data are presented as the mean \pm SD. Differences between means were determined by t-test and ANOVA. The Tukey's test was used to compare differences amongst means. Differences were defined as significant at $\mathrm{p}<0.05$.

\section{RESULTS AND DISCUSSION}

Antimicrobial activity of pure geraniol and linalool as assessed with the disc diffusion method

In the present study, the diameter of the inhibition zone was used to evaluate the antimicrobial efficiency of geraniol and linalool against 


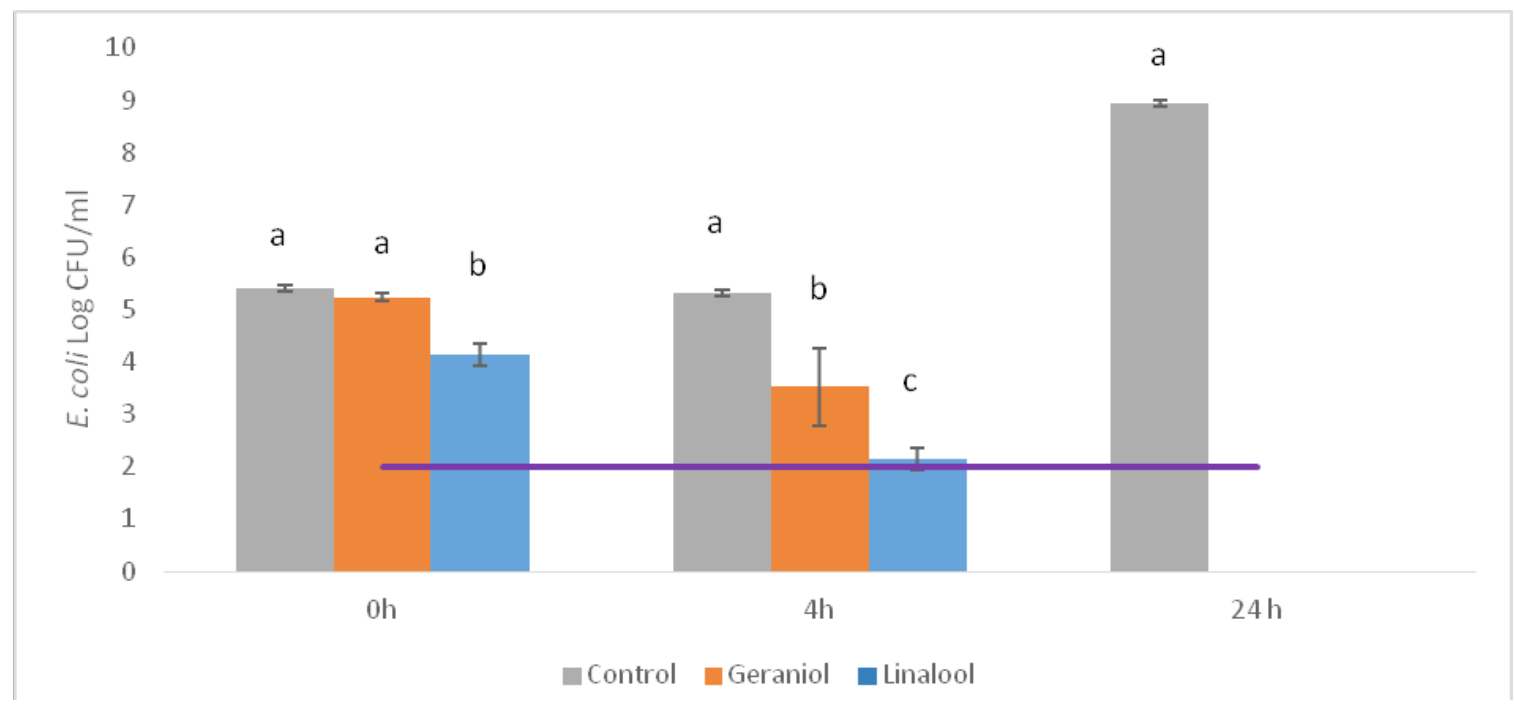

Fig. 1. Effect of geraniol and linalool nanoemulsions at a concentration of $4000 \mathrm{ppm}$ on $E$. coli counts in meat simulation medium at different time intervals. Each point represents the mean \pm standard deviation. Different letters for each time point denote statistically significant differences $(\mathrm{p}<0.05)$. The line represents the detection limit. E. coli counts for geraniol and linalool nanoemulsions were below the detection limit.

E. coli K12, L. innocua and Ps. lundensis. Results in Table 1 show that both antimicrobials had a potent antibacterial effect against all bacteria tested. Linalool was most effective against $E$. coli $(18.32$ $\pm 1.17 \mathrm{~mm}$ ) whereas geraniol was most effective against $L$. innocua $(13.83 \pm 0.35 \mathrm{~mm})$. Similar antimicrobial activity has been found for carvacrol against 0157 and non-0157 E. coli (Stratakos et al. 2017).

\section{Characterisation of nanoemulsions}

Results showed that both geraniol and linalool could be encapsulated in nanoemulsions. Characterisation with dynamic light scattering showed that the geraniol and linalool nanoemulsions had a mean diameter of 68.22 \pm 2.46 and $173.59 \pm 4.15 \mathrm{~nm}$, respectively. The polydispersity index for geraniol and linalool was also determined and was found to be $0.20 \pm$ 0.005 and $0.24 \pm 0.006$, respectively. Landry et al. (2014) found similar results when they prepared nanoemulsions loaded with carvacrol with a mean droplet diameters of approx. $100 \mathrm{~nm}$, as determined by dynamic light scattering.

Effect of geraniol and linalool nanoemulsions on the survival of $E$. coli in meat simulation medium.

Results presented in Figure 1 show that both nanoemulsions were effective against $E$. coli $\mathrm{K} 12$ in the meat simulation medium. The linalool nanoemulsion resulted in a significant reduction in E. coli counts immediately after treatment (approx. $1 \log \mathrm{CFU} / \mathrm{ml}$ ). At $4 \mathrm{~h}$ incubation the linalool nanoemulsion reduced counts by 3.17 log whereas the geraniol nanoemulsion by $1.8 \mathrm{log}$ CFU ml. Both nanoemulsions were able to reduce E. coli counts below the detection limit which corresponds to $>3$ log reduction in $E$. coli counts, compared to the initial inoculum (approx. $5 \mathrm{log}$ $\mathrm{CFU} / \mathrm{ml}$ ), after $24 \mathrm{~h}$ incubation.

According to Barbosa et al., (2009) oregano, thyme, basil, marjoram, lemongrass, ginger, and clove essential oils showed a significant reduction against $S$. aureus, L. monocytogenes, E. coli, and Salmonella Enteritidis in vitro. However, when these pathogens were artificially inoculated on irradiated beef meat resulted in a non-statistically significant reduction in the pathogen counts $(<1$ $\log$ CFU/g). Revealing loss of their activity when used in real food systems.

Effect of geraniol and linalool nanoemulsions on the survival of L. innocua in meat simulation medium.

Results presented in Figure 2 show, in this case as well, that both nanoemulsions were effective against $L$. innocua in the meat simulation medium. Untreated samples (control) grew rapidly, as expected. However, in this case both nanoemulions proved to be equally effective with L. innocua counts not differing significantly (p $>0.05$ ) throughout the incubation. At $24 \mathrm{~h}$ both 


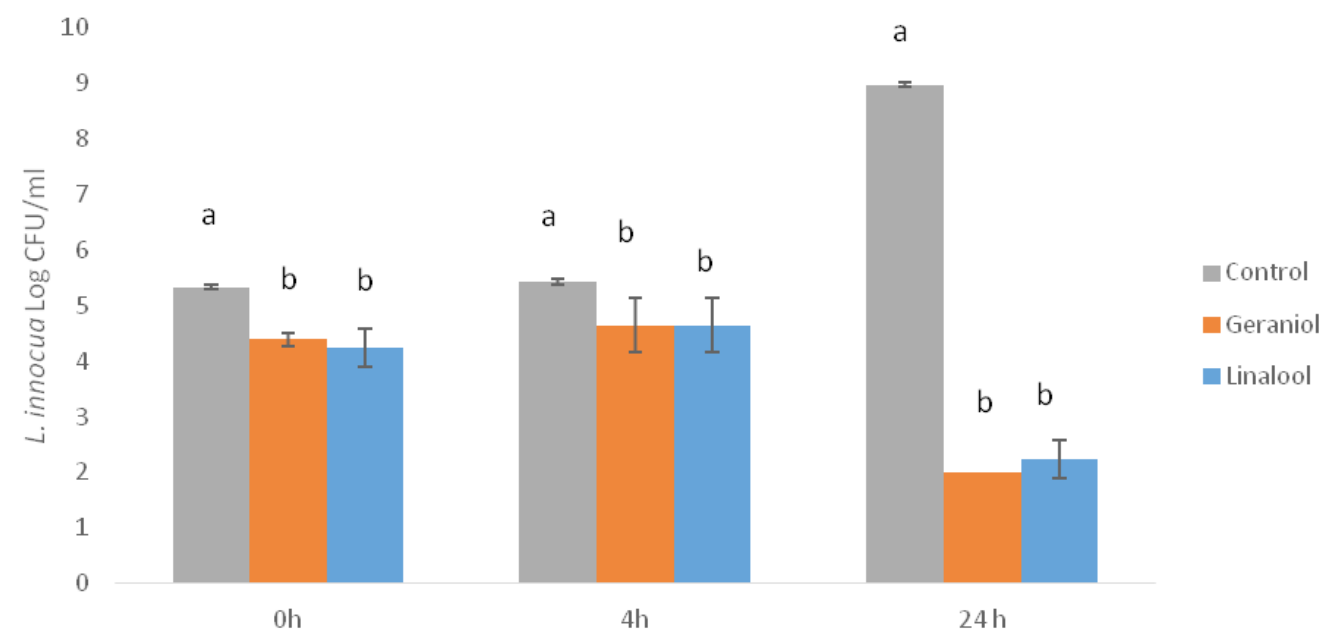

Fig. 2. Effect of geraniol and linalool nanoemulsions at a concentration of $4000 \mathrm{ppm}$ on L. innocua counts in meat simulation medium at different time intervals. Each point represents the mean \pm standard deviation. Different letters for each time point denote statistically significant differences $(\mathrm{p}<0.05)$.

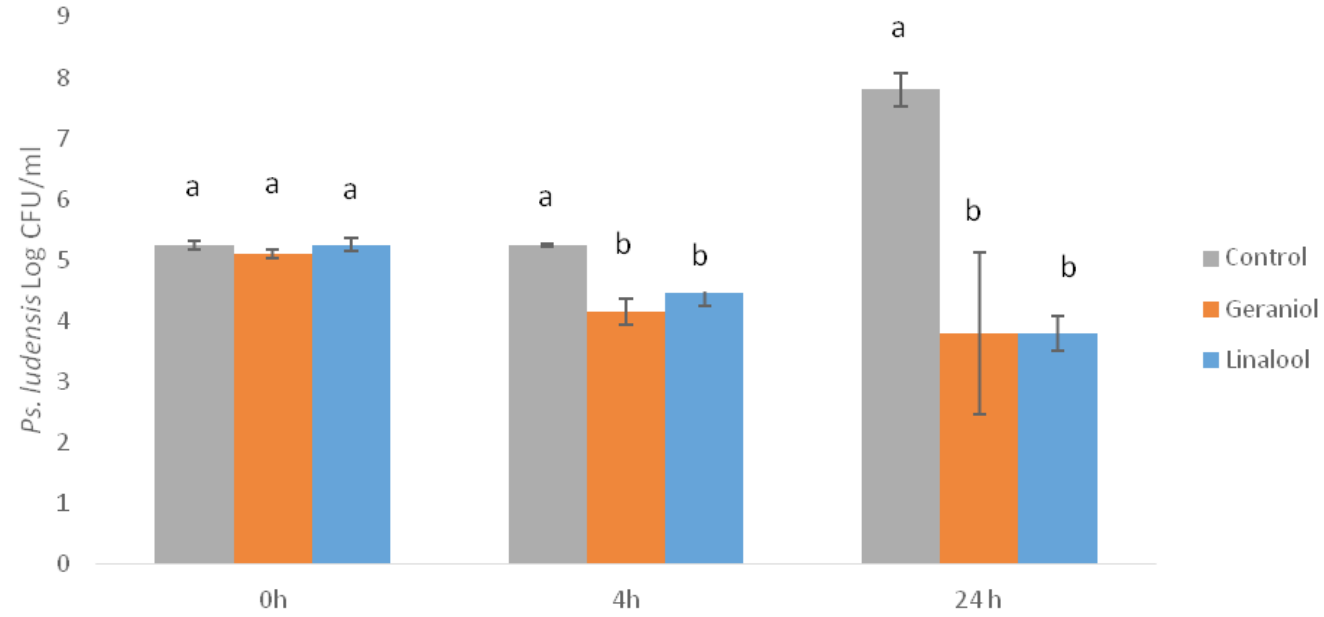

Fig. 3. Effect of geraniol and linalool nanoemulsions at a concentration of $4000 \mathrm{ppm}$ on Ps. lundensis counts in meat simulation medium at different time intervals. Each point represents the mean \pm standard deviation. Different letters for each time point denote statistically significant differences $(p<0.05)$.

nanoemulsions reduced counts by approx. 3 logs, compared to the initial inoculum (approx. $5 \mathrm{log}$ $\mathrm{CFU} / \mathrm{ml}$ ).

Similar results were found in the study of Maté et al., (2016) in which the antibacterial effect of nanoemulsions loaded with the natural compound d-limonene against Listeria monocytogenes in tryptic soy broth growth medium, chicken broth, and vegetable cream, was investigated. The counts of L. monocytogenes decreased approx. 3 log cycles in all growth media tryptic soy broth growth medium, chicken broth, and vegetable cream. The study also showed greater effectiveness when applying D-limonene in form of nanoemulsion than when applying it directly,

Effect of geraniol and linalool nanoemulsions on the survival of Ps. lundensis in meat simulation medium.

Ps. lundensis is one of the most prominent species of Pseudomonas that cause spoilage of meat during storage. Figure 3 presents the effect of geraniol and linalool nanoemulsions against this spoilage microorganism. In this case, there was no immediate effect on Ps. Lundensis with the counts of all three treatments not differing significantly ( $p$ > 0.05). After $4 \mathrm{~h}$ incubation the geraniol and linalool nanoemulsion treatments were significantly lo- 
wer $(\mathrm{p}<0.05)$ compared to the control $(4.15$ and $4.45 \log \mathrm{CFU} / \mathrm{ml}$, respectively). After $24 \mathrm{~h}, P s$. Lundensis counts were further reduced to 3.79 and $3.80 \log \mathrm{CFU} / \mathrm{ml}$ for the geraniol and linalool nanoemulsions, respectively.

In summary, two food-grade nanoemulsions loaded with geraniol or linalool were tested for their efficacy against $E$. coli K12, L. innocua and $P$ s. lundensis in a meat simulation system. Both of the nanoemulsions fabricated and tested in this study showed very promising results. Geraniol and linalool nanoemulsions were able to significantly reduce $E$. coli and $L$. innocua counts. Also, although both nanoemulsions were able to significantly reduce Ps. Lundensis, this bacterium appeared to be less susceptible to the action of the nanoemulsions compared to E. coli K12 and L. innocua. Nanoemulsion technology could potentially help resolve issues of solubility and stability of hydrophobic antimicrobials in the food industry. With greater understanding of the system as a whole, they may find a broad range of applications in food preservation and food safety. Therefore, it would be useful in future studies to test the efficacy of the antimicrobial nanoemulsions developed here in real food systems to establish their range of efficacy.

\section{REFERENCES}

1. Barbosa LN, Rall VLM, Fernandes AAH, Ushimaru PI, da Silva Probst I, Fernandes JrA (2009). Essential oils against foodborne pathogens and spoilage bacteria in minced meat. Foodborne pathogens and disease, 6, 725-728.

2. Burt S (2004). Essential oils: their antibacterial properties and potential applications in foods-a review. International journal of food microbiology, 94 223-253.

3. Manya M (2008). Essentials of Infectious Disease Epidemiology. Essential Public Health. United StatesISBN-10:0763738786.

4. Chang Y, McLandsborough L, McClements DJ (2013). Physicochemical properties and antimicrobial efficacy of carvacrol nanoemulsions formed by spontaneous emulsification. Journal of agricultural and food chemistry, 61, 8906-8913.

5. Landry KS, Chang Y, McClements DJ, McLandsborough L (2014). Effectiveness of a novel spontaneous carvacrol nanoemulsion against Salmonella enterica Enteritidis and Escherichia coli 0157: H7 on contaminated mung bean and alfalfa seeds. International journal of food microbiology, 187, 15-21.

6. Maté J, Periago PM, Palop A (2016). Combined effect of a nanoemulsion of d-limonene and nisin on Listeria monocytogenes growth and viability in culture media and foods. Revista de Agaroquimica y Tecnologia de Alimentos, 22,146-152.

7. Settanni L, Palazzolo E, Guarrasi V, Aleo A, Mammina C, Moschetti G, Germanà MA (2012). Inhibition of foodborne pathogen bacteria by essential oils extracted from citrus fruits cultivated in Sicily. Food Control, 26, 326-330.

8. Xiao D, Gömmel C, Davidson P M, Zhong Q (2011). Intrinsic Tween 20 improves release and antilisterial properties of co-encapsulated nisin and thymol. Journal of Agriculture and Food Chemistry, 59, 9572-9580.

9. Zahi MR, Liang $H$, Yuan $Q$ (2015. Improving the antimicrobial activity of d-limonene using a novel organogel-based nanoemulsion. Food Control, 50, 554559.

10. Zhang Y, Liu X, Wang Y, Jiang P, Quek S (2016). Antibacterial activity and mechanism of cinnamon essential oil against Escherichia coli and Staphylococcus aureus. Food Control, 59, 282-289. 